101

\section{Amyotrophic Lateral Sclerosis (ALS) - Not Just a Motor Disease? Isolated Bitter and Sweet Taste} Loss in ALS

\author{
Ahmed A Ashary, MD'; Dev N Patel ; and \\ Alan R Hirsch, $M D^{3}$ \\ ${ }^{1}$ St. Georges, University of London Medical Programme \\ at University of Nicosia, Cyprus \\ ${ }^{2}$ Aureus University School of Medicine, Aruba \\ ${ }^{3}$ Smell and Taste Treatment and Research Foundation, \\ Chicago, Illinois
}

ABSTRACT: Study Objective: Specific taste quality deficits in ALS has not heretofore been described.

METHOD: Case Study: A 71 year old right handed female presented with a two year course of progressive reduction in strength in her hands, arms and legs with difficulty tying shoe laces, opening jars, writing and walking. She described nocturnal muscle spasms involving all extremities. Gradually, over eight months prior to presentation, all food began to taste bad and horribly bitter. Associated with no appetite and a seven pounds weight loss.

RESULTS: Abnormalities in Neurological examination: Cranial Nerve (CN) examination: CN IX and X: Gag absent bilaterally. Motor examination: Bulk: atrophy in thenar and hypothenar eminences and intrinsics in both upper extremities. Percussion induced fasciculation and myotonia in both shoulders and arms. Fasciculation of tongue with percussion myotonia of tongue. Strength: Intrinsic $4 / 5$ in both upper extremities, $3 / 5$ in abductor policis brevis bilaterally, $3 / 5$ right gastrocnemius soleus, 4/5 bilateral anterior tibialis. Drift testing: left abductor digiti minimi sign. Gait: Heel and toe walking unstable with circumduction of left leg. Tandem gait unstable. Cerebellar: Holmes rebound phenomena positive in the left upper extremity. Deep tendon reflexes: 1+ left brachioradialis. $1+$ left triceps. $3+$ right ankle jerks. 0 left ankle jerk. Positive jaw jerk. Chemosensory Testing: Normosmia to: Alcohol Sniff Test (46), Pocket Smell Test (3/3) and Retronasal Smell Index (9). Taste Quadrant Testing: ageusia in the palate to sodium chloride and citric acid. Ageusia throughout the palate, tongue and whole mouth to sucrose and quinine hydrochloride. Fungiform papillae count: left 18, right 20 (normal). Lip biopsy (normal). MRI: T2 flair in bilateral corticospinal tracts, left greater than right in the spinal cord and the brain. EMG: fibrillation, positive waves with fasciculation in all four extremities. Voluntary contraction with polyphasic unstable motor unit action potentials.

CONCLUSION: While Lang found no taste loss in ALS (Lang, 2011), Pelletier found reduction in intensity of taste to all modalities in different sectors of the tongue, but paradoxically demonstrated normogeusia in whole mouth taste perception (Pelletier, 2013). Pathological specimens of those with ALS revealed degeneration in the nucleus parabrachialis medialis and tractus trigeminothalamicus dorsalis (Oyanagi, 2015), suggesting that taste deficit may be due to central white matter abnormalities. Sweet taste is localized in the most posterior and rostral aspect of the right insular cortex, immediately adjacent to bitter (Prinster, 2017), suggesting a neighborhood effect phenomena. Weight loss in ALS may be due to sensory distortion and secondary impairment of appetite. It would be worthwhile to investigate those with ALS for evidence of otherwise overlooked gustatory deficits, correction of which may improve appetite and nutritional state.

102

\section{Extracurricular Activity Involvement and Depression Among High School Students}

\author{
Alex Waler'; and Lindsay Taliaferro, Ph. D. \\ M.P.H. CHES ${ }^{2}$ \\ ${ }^{1}$ M.D. Candidate, Class of 2021, University of Central \\ Florida College of Medicine, Orlando, Florida \\ ${ }^{2}$ Assistant Professor of Medicine, Population Health, \\ University of Central Florida College of Medicine, \\ Orlando, Florida
}

ABSTRACT: Introduction: While physical activity has been shown to promote positive mental health and aid in treatment, the association between involvement in other activities and depressive symptoms among adolescents remains unclear. We sought to fill a gap in the literature by examining relationships between involvement in sports, art, and leadership activities during high school and depressive symptoms among adolescents. Hypothesis: We hypothesized that all types of involvement would be associated with lower levels of depressive symptoms.

METHODS: We performed a secondary data analysis using the 2016 Minnesota Student Survey, a population-based survey of students in grades 9 and $11(\mathrm{~N}=81,885)$. Descriptive sample statistics (e.g., frequencies) and chisquare tests, stratified by sex, were used to examine relationships between depressive symptoms and involvement in different extracurricular activities among males and females. Analyses were performed using SPSS.

RESULTS: For males and females, involvement in sports was associated with lower levels of depression, compared to non-involvement. Compared to males who did not participate in arts $(16.6 \%)$, those who did were significantly more likely to report depressive symptoms 
$(21.2 \%)$. In addition, females involved in leadership activities were significantly less likely to report depressive symptoms $(21.9 \%)$ than those who were not involved in these activities (28.7\%). Consistent with previous research, females were more likely to report depressive symptoms than males. Females also were more likely to participate in arts and leadership activities.

CONCLUSION: For males and females, sports participation, and for females, involvement in leadership activities, may represent protective factors against depressive symptoms during adolescence. However, clinicians might consider inquiring about depressive symptoms among adolescent males involved in art-related activities.

\section{ORIGINAL CITATION:}

Waler A, Taliaferro L. Extracurricular Activity Involvement and Depression Among High School Students. Oral presentation and poster presentation, 10th Annual FIRE Conference, Orlando, Florida. February 21, 2019.

\section{3}

\section{Atypical Progressive Bulbar Palsy presenting with Dropped Head}

Alexander Carvajal-González, $\mathrm{MD}, \mathrm{PhD}^{\prime}$; and Antonio H Iglesias, $\mathrm{MD}^{2}$

${ }^{1}$ Assistant Professor, School of Medicine, Universidad El Bosque, Bogotá, Colombia

${ }^{2}$ Associate Professor, Neurology Department, Loyola University Medical Center, Chicago, USA.

ABSTRACT: Introduction: Typical amyotrophic lateral sclerosis (ALS) presents on neurological examination with specific signs of upper and lower motor neuron degeneration (Brooks et al, 1995), which can account for $85 \%$ of patients with ALS (Turner and Talbot, 2013). There are different types of clinical presentations, including progressive bulbar palsy (PBP), Limb-onset ALS, progressive muscular atrophy (PMA) and upper motor neuron (UMN) predominant ALS. PBP has mainly brainstem signs. There are a few case reports of dropped head syndrome in ALS, mainly in patients with the limb involvement variant.

\section{METHODS: Case report}

RESULTS: A 56 year old right-handed male, presented to the clinic with four months of dysphagia to liquids and solids, neck pain and progressive neck weakness causing constant drop head. No dysarthria or other neurological symptoms, no dyspnea. Neurological examination: Cranial Nerve (CN) CN XII: Nasal voice, bilateral atrophy of the tongue with tremor and fasciculations. Motor: Diffuse atrophy and decreased tone of the sternocleidomastoid and trapezii bilaterally, strength: $2 / 5$ in neck flexors and extensors. Sensory: Hypoesthesia of the tongue. The rest of his neurological examination was normal. Labs: Routine blood work, thyroid function tests, collagen vascular work-up, and protein electrophoresis were normal. Creatine Phosphokinase (CPK) and Acetylcholine Receptor Antibodies (AChR Ab) were negative. Brain and Spinal Cord MRI: Showed mild brainstem, cerebellar and cervical spinal atrophy.

CONCLUSIONS: Patients with ALS initially present with symptoms localized to the limbs or bulbar muscles. A very small percentage 1-2\% of ALS patients had neck muscle weakness with head drop (Jokelainen et al, 1977; GourieDevi et al, 2003). However, in all the previously reported cases, the patients had limb involvement at the time of presentation which was absent in this case, and the head drop occurred after the onset of symptoms (Lange et al, 1986; Katz et al; 1996). Dropped head syndrome can be seen in inflammatory myopathies, myasthenia gravis, facioscapulohumeral muscular dystrophy, spinal muscular atrophy, nemaline myopathy and carnitine deficiency (Umapathy et al, 2003) but ALS should also be considered in patients with atypical presentations.

\section{4}

Long-term Efficacy of Lurasidone in Antipsychoticnaïve vs. Antipsychotic-exposed Adolescents with Schizophrenia: Analysis of a Two-Year Study

Christoph U. Correll, $M D^{\prime}$; Robert Goldman, $P h D^{2}$; Michael Tocco, PhD ${ }^{2}$; Jay Hsu, $P h D^{2}$; and Andrei Pikalov, $M D, P h D^{2}$

${ }^{1}$ The Zucker Hillside Hospital, Department of Psychiatry, Northwell Health, Glen Oaks, NY, USA; Hofstra Northwell School of Medicine, Department of Psychiatry and Molecular Medicine, Hempstead, NY, USA; and Charité Universitätsmedizin, Department of Child and Adolescent Psychiatry, Berlin, Germany ${ }^{2}$ Sunovion Pharmaceuticals Inc, Fort Lee, NJ and Marlborough, MA

ABSTRACT: Background: Early-onset schizophrenia is characterized by greater severity and more functional impairment than adult-onset schizophrenia. Few studies have prospectively evaluated short- or long-term antipsychotic efficacy in treatment-naïve (vs. previously treated) firstepisode schizophrenia. The aim of this post-hoc analysis was to evaluate the long-term efficacy of lurasidone in antipsychotic-naïve adolescents with schizophrenia.

METHOD: Patients aged 13-17 years with schizophrenia were randomized to 6 weeks of double-blind (DB), fixed-dose treatment with lurasidone $(40 \mathrm{mg} /$ day or $80 \mathrm{mg}$ /day) or placebo. Six-week completers were eligible to enroll in an open-label (OL), flexible dose 2-year 